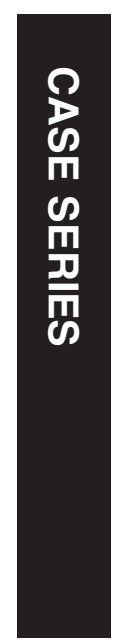

${ }^{1}$ Department of Ophthalmology, Royal Victoria Hospital, Belfast, UK

${ }^{2}$ Department of Ophthalmology, Mater Hospital, Belfast, UK

${ }^{3}$ Northern Ireland Regional Genetics Department, Belfast City Hospital, Belfast Health \& Social Care Trust, Belfast, UK

${ }^{4}$ Department of Eye and Vision Science, Institute of Ageing and Chronic Disease, University of Liverpool, Liverpool, UK

Correspondence: S Chamney, Department of Ophthalmology, Royal Victoria Hospital, 274 Grosvenor Road, Belfast BT12 6BA, Antrim, UK Tel: +44 (0)2890240503; Fax: +44 (0)2890330744. E-mail: sarahchamney@ gmail.com

Received: 15 September 2014 Accepted in revised form: 8 May 2015

Published online:

16 October 2015

This case series was presented as a poster at the Irish College of Ophthalmology Conference in 2008.

\title{
The ocular phenotype of stiff-skin syndrome
}

\begin{abstract}
Purpose Stiff skin syndrome (SSS; MIM\#184900) is a rare autosomal dominantly inherited Mendelian disorder characterised by thickened and stone-hard indurations of the skin, mild hypertrichosis, and limitation of joint mobility with flexion contractures. It is autosomal dominant with high penetrance and results from mutations in the fibrillin 1 (FBN1; MIM*134797) gene. Here we present the associated ocular phenotype in a two generation nonconsanguineous Northern Irish family.

Methods The affected patients underwent complete ophthalmic and orthoptic assessment and genetic testing.

Results All three patients had ophthalmoplegia of varying degrees. Direct sequencing of the FBN1 gene detected a heterozygous pathogenic mutation (c.4710G > C; p.Trp1570Cys) in all affected patients.

Conclusions This is the first report of ophthalmoplegia in association with SSS. Eye (2016) 30, 156-159; doi:10.1038/eye.2015.183; published online 16 October 2015
\end{abstract}

\section{Introduction}

Stiff skin syndrome (SSS; MIM\#184900) is a rare Mendelian autosomal dominant disorder characterised by thickened, stone-hard indurations of the skin, mild hypertrichosis, and limitation of joint mobility with flexion contractures. It was first reported in $1971 .{ }^{1}$ It is caused by pathogenic mutations in the Arg-Gly-Asp (RGD) sequenceencoding domain of fibrillin 1 (FBN1) gene that mediates intergrin binding. ${ }^{2}$ No previous descriptions of the ocular phenotype in SSS have been published and herein we report the ocular findings of an affected family.

\section{Case reports}

Three patients (a mother and her two daughters) were diagnosed with SSS at the Northern Ireland Regional Genetics Service, Belfast. There was an
S Chamney ${ }^{1}$, B Cartmill' ${ }^{1}$ O Earley²,

$\checkmark$ McConnell $^{3}$ and CE Willoughby ${ }^{4}$

unaffected son from the sibship. Diagnosis was confirmed at the molecular level using direct sequencing; all affected family members had a heterozygous $F B N 1$ pathogenic gene mutation (c.4710G > C; p.Trp1570Cys). Ophthalmic and orthoptic examinations were subsequently completed.

\section{Case 1: Proband (54-year-old female)}

This patient wore glasses from the age of 5 years and had been diagnosed with right amblyopia, which was treated with occlusion therapy. Aged 51 years, she was diagnosed with bilateral posterior subcapsular cataracts and had uncomplicated phacoemulsification and intraocular lens implantation. The left eye was unexpectedly myopic post-operatively and was corrected by a LASIK (Laser Assisted in situ Keratomileusis) procedure.

She had been diagnosed with flexion contractures of fingers and elbows at 7 years of age and was found to have thickened skin on her hands and arms. By the age of 11 years the patient noticed stiffening/clawing of her hands, which by the age of 15 years was affecting her employment as a seamstress. By the age of 47 years predominately the distal and proximal interphalangeal joints of the hands were affected with evidence of cutaneous nodules. There has been some evidence of mild nail dysplasia. She has had respiratory symptoms and in particular shortness of breath from age of 33 years and has been under the care of a respiratory physician.

Her best-corrected visual acuity was 6/18 in both eyes. The left cornea showed evidence of previous LASIK. Both eyes showed well-centred stable posterior chamber IOLs. The posterior pole was normal in both eyes. Cover testing revealed orthophoria for distance and 10 prism dioptres of exophoria at near. The extraocular movements of both eyes were abnormal with restriction of elevation, depression, abduction, and adduction (Figure 1). 


\section{Case 2 (23-year-old female)}

This patient was the first daughter of the proband and reported difficulty looking up and to the left. She tended to move her head instead of her eyes when looking to the side. She had worn glasses from the age of 8 years. She was noted to have fissured palms at birth, generalised stiff skin, flexion contractions at both elbows, tight hard skin on hands and feet. She toe walked and had Achilles tendon lengthening procedures at ages 8 and 14 .

Her best-corrected visual acuity was 6/6 right eye and 6/5 left eye and N5 in both eyes. Her anterior and posterior chamber examination was normal. Cover testing revealed 1 prism dioptre of exophoria for distance with 10 prism dioptres of exophoria for near. Extraocular movements were restricted in upgaze and in lateral gaze (Figure 2).

\section{Case 3 (22-year-old female)}

This patient who was the second daughter of the proband reported difficulty looking up and to the sides. She had worn glasses from 4 years of age. She had generalised stiff-skin flexion contractions of both elbows, a history of toe walking for which bilateral Achilles tendon release procedures were completed at age 7 years.

Her best-corrected visual acuity was $6 / 6$ right eye and 6/9 left eye. Anterior segment examination was normal. The crystalline lens in the right eye was normal however in the left eye showed few refractile dot-like opacities in the nucleus. There was no phacodonesis or subluxation. Posterior segment examination was normal. Cover testing revealed 1 prism dioptre of exophoria for distance with 12 prism dioptres of exophoria for near. Extraocular movements were restricted in all positions of gaze (Figure 3).

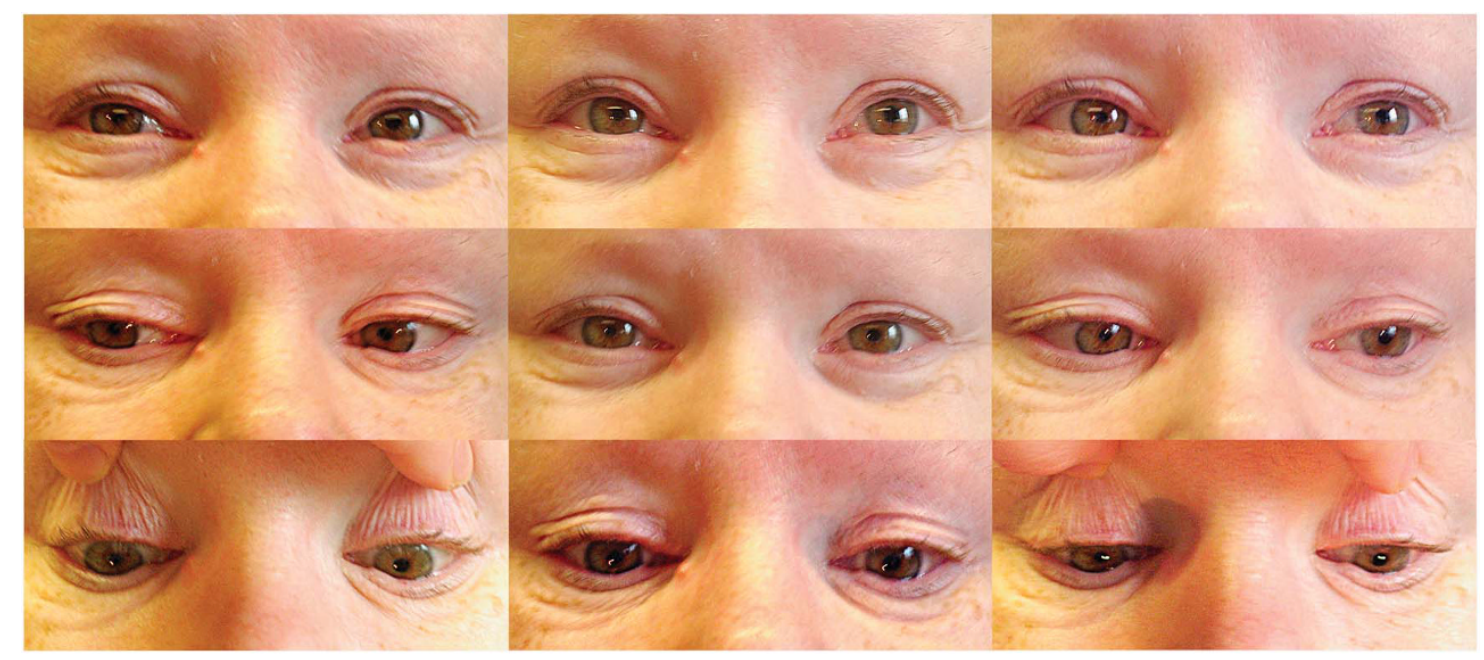

Figure 1 Patient 1 eight positions of gaze.

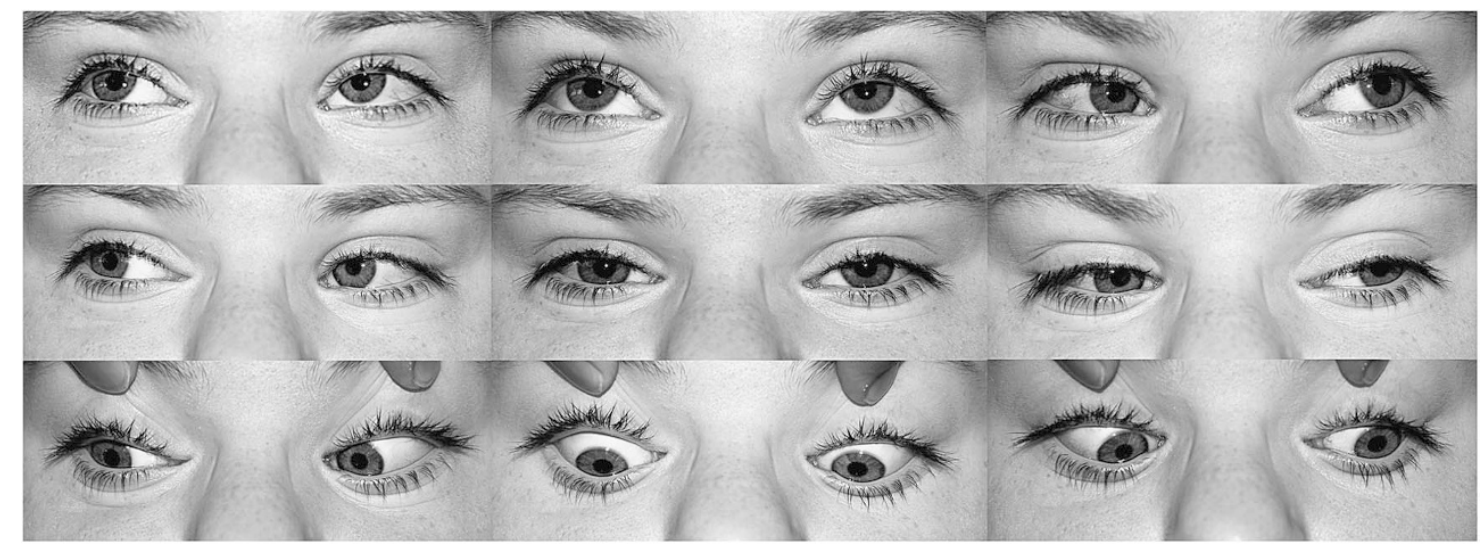

Figure 2 Patient 2 eight positions of gaze. 


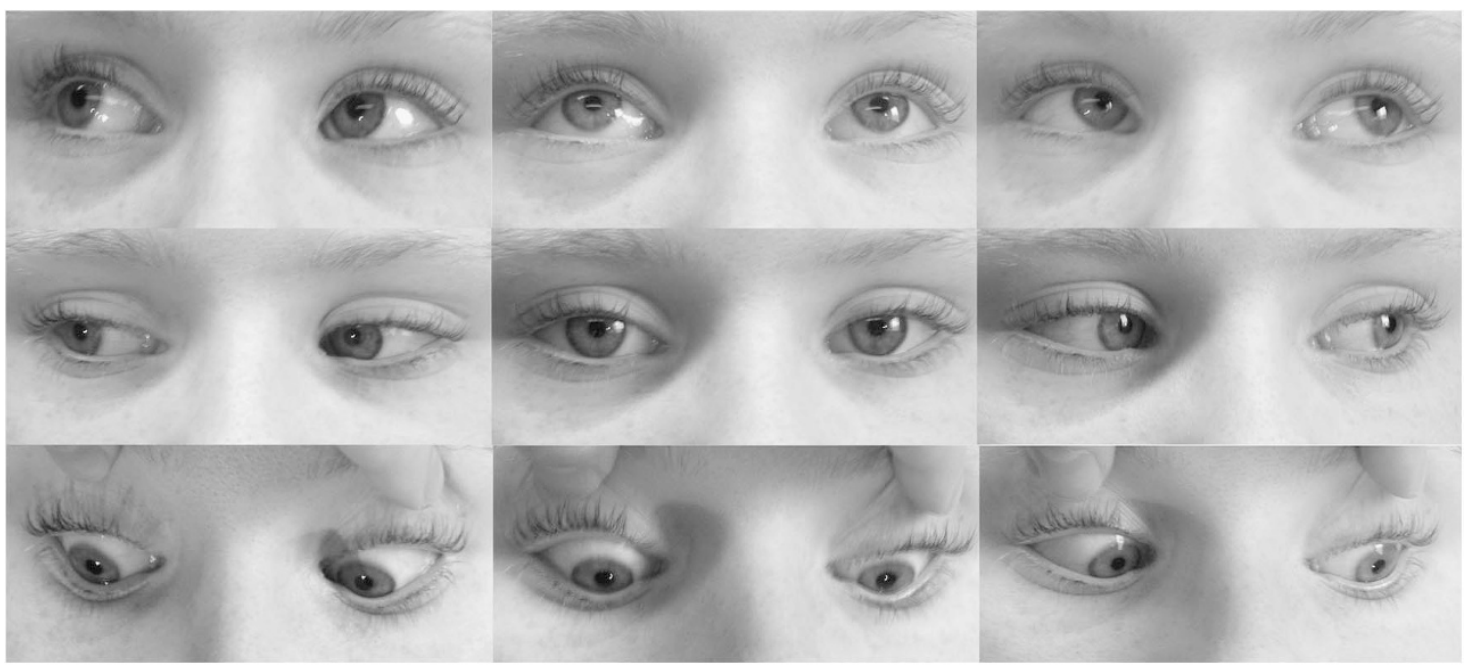

Figure 3 Patient 3 eight positions of gaze.

\section{Discussion}

SSS usually presents with stony hard thick skin that limits joint mobility congenitally or before the age of 6 years. ${ }^{3}$ The hard skin is bound firmly to the underlying tissues usually over the entire body, with the most common areas being the buttocks, thighs and shoulder areas that limits joint mobility and cause flexion contractures. Also cutaneous nodules that predominantly affect DIP joints and diffuse entrapment neuropathy are associated. Rarely SSS can be associated with scoliosis, restrictive pulmonary changes (as observed in the proband), focal

lipodystrophy, hypertrichosis, muscle weakness, growth retardation, and /or relative short stature. The disease is felt to be slowly progressive. It is a rare autosomal dominantly inherited mendelian disorder with $<40$ reported cases in the literature. Fibrillin is essential in the mechanical strength of many tissues and contains binding site for integrin receptors which induce intracellular adaptations in response to extracelluar changes. Mutations in FBN1 result in SSS and Marfan syndrome. ${ }^{2}$ The pathogenic mutations identified in SSS are in the Arg-Gly-Asp (RGD) sequence-encoding domain of fibrillin-1 that mediate integrin binding, resulting in a fibrotic phenotype. ${ }^{4}$ There is a wide phenotype variation described in the literature. The milder cases may represent a localised condition due to mosaic forms of SSS. Treatment for SSS is usually supportive with physiotherapy.

The ocular phenotype in this family with SSS consisted of a mechanical restriction of extraocular movement consistent with fascial shortening within the orbit. This is most likely due to the same pathophysiology as the limb contractions and dermatological findings; giant collagen fibril formation without inflammation in the extraocular fascia network. ${ }^{5,6}$ Ophthalmoplegia has not been documented in previous case reports of SSS. ${ }^{7-13}$

Summary

What was known before

- SSS is a rare autosomal dominant Mendelian disorder with high penetrance.

- It is caused by pathogenic mutations in the Arg-Gly-Asp sequence-encoding domain of the Fibrillin 1 (FBN1) gene.

What this study adds

- Restrictive ophthalmoplegia is part of the SSS ocular phenotype and ophthalmic assessment is recommended.

- Patients with this restrictive ophthalmoplegia should undergo systemic evaluation for SSS.

\section{Conflict of interest}

The authors declare no conflict of interest.

\section{References}

1 Esterly NB, McKusick VA. Stiff skin syndrome. Pediatrics 1971; 47: 360-369.

2 Loeys BL, Gerber EE, Riegert-Johnson D, Iqbal S, Whiteman P, McConnell V et al. Mutations in fibrillin-1 cause congenital scleroderma: stiff skin syndrome. Sci Transl Med 2010, 2: 23ra20.

3 Liu T, McCalmont T, Frieden I, Williams M, Connolly K, Gilliam A. The stiff skin syndrome: Case series, differential diagnosis of the stiff skin phenotype and review of the literature. Arch Dermatol 2008; 144: 1351-1359.

4 Olivieri J, Smaldone S, Ramirez F. Fibrillin assemblies: extracellular determinants of tissue formation and fibrosis. Fibrogenesis Tissue Repair 2010; 3: 24.

5 McCalmont T, Gilliam A. A subcutaneous lattice- like array of thick collagen is a clue to the diagnosis of stiff skin syndrome. J Cutan Pathol 2012; 39: 2-4. 
6 Fidzianska A, Jablonska S. Congenital fascial dystrophy: abnormal composition of the fascia. J Am Acad Dermatol 2000; 43: 797-802.

7 Gilaberte Y, Saenz-de-Santamaria MC, Garcia-Latasa FJ, Gonzalez-Mediero I, Zambrano A. Stiff skin syndrome: a case report and review of the literature. Dermatology 1995; 190: $148-151$.

8 Pages ON, Maliszewicz P, Lefebvrel F, Poli-Merol ML, Morville P. Visceral involvement in stiff skin syndrome. Pediatr Dermatol 2007; 24: 327.

9 Amorim AGF, Aide MK, Duraes SMB, Rochael MC. Stiff skin syndrome: a case report. An Bras Dermatol 2011; 86(4 Suppl 1): S178-S181.
10 Azevedo V, Serafini S, Werner B, Muller C, Franchini C, Morais R. Stiff skin syndrome versus scleroderma: a report of two cases. Clin Rheumatol 2009; 28: $1107-1111$.

11 Geng S, Lei X, Toyohara, Zhan P, Wang J, Tan S. Stiff skin syndrome. J Eur Acad Dermatol Venereol 2006; 20: 729-732.

12 Jablonska S, Blaszczyk M. Scleroderma-like indurations involving fascias: an abortive form of congenital fascial dystrophy. Pediatr Dermatol 2000; 17: 105-110.

13 Caspary P, Costa F, Souza PR, Dupuia RP, de Almeida HL. Familial localized stiff skin syndrome. Int J Dermatol 2010; 49: 843-845. 